Received: 2019.03 .15 Accepted: 2019.03.28 Published: 2019.06 .08

\title{
Coronary Artery Bypass Grafting in a Patient with Situs Inversus Totalis
}

1 Department of Cardiac Surgery, European Medical School Oldenburg-Groningen, Carl von Ossietzky University Oldenburg, Oldenburg, Germany

2 Department of Cardiac Surgery, E.A. Vagner Perm State Medical University, S.G. Sukhanov Federal Center of Cardiovascular Surgery, Perm, Russian Federation
Data Collection B Statistical Analysis $C$ Data Interpretation D Manuscript Preparation E

Literature Search F Funds Collection G

\author{
ABCDEF 1 Konstantin Zhigalov \\ BEF 2 Danil Ponomarev \\ BEF 2 Artem Sozkov \\ BEF 2 Bakitbek Kadyraliev \\ BEF 1 Jerry Easo \\ ABCDEF 1 Alexander Weymann
}

Corresponding Author: Conflict of interest:
Konstantin Zhigalov, e-mail: konstantin.zhigalov@yahoo.com None declared

\begin{abstract}
Patient: Male, $\mathbf{5 0}$
Final Diagnosis: Coronary artery disease in a patient with situs inversus totalis

Symptoms: Angina pectoris

Medication: -

Clinical Procedure: Coronary artery bypass grafting

Specialty: Cardiac surgery

Objective: Rare disease

Background: We report a rare case of total arterial coronary artery bypass grafting (CABG) in a patient with situs inversus totalis (SIT). SIT is a rare variant of biologically normal anatomy, in which the main internal organs are mirrored compared to normal localization.

Case Report: A 50-year-old patient with SIT and severe coronary stenosis of the left anterior descending artery (LAD) and the ramus circumflexus (RCX) was admitted to our hospital. CABG was performed promptly using 2 conduits. After medial sternotomy, both mammary arteries were isolated by the skeletal method. Next, the cannulation of the aorta and the physiologically right atrium was performed. Custodiol cardioplegia was performed antegrade in a single shot. The left internal mammary artery (LIMA) was anastomosed to the LAD and the right internal mammary artery (RIMA) to the RCX. The aortic clamping time was 29 minutes. The operation was completed without complications. The patient was extubated 6 hours after surgery. On the first postoperative day, the patient was transferred to a regular ward. Postoperative therapy was performed without complications.

Conclusions: $\quad$ Surgical correction is indicated for many patients with SIT in combination with coronary heart disease. Performing a CABG operation on these patients requires the operating team to have certain skills. Surgeons face a number of problems and issues: preoperative preparation of the patient, the position of the surgeon to the left or right during the operation, the choice of conduit for anastomosis, and the tactics of the operation.
\end{abstract}

MeSH Keywords: Coronary Artery Bypass • Coronary Disease • Dextrocardia

Full-text PDF: https://www.amjcaserep.com/abstract/index/idArt/916250 


\section{Background}

We report a case of total arterial coronary artery bypass grafting in a patient with situs inversus totalis. Situs inversus totalis is a rare variant of biologically normal anatomy, in which the main internal organs are mirrored compared to normal localization. The position of the heart is determined by the localization of the atria - the morphologically right atrium is on the left and the morphologically left is on the right [1].

Dextrocardia in combination with situs inversus is a rare congenital anomaly, with a frequency of 1: 10000 [2]. Cardiac abnormalities are rare in patients with situs inversus totalis, whereas abnormalities associated with isolated dextrocardia occur more frequently.

The incidence of coronary artery disease in these patients is similar to that of the general population. Coronary artery bypass grafting $(C A B G)$ operations in this cohort of patients are associated with certain difficulties and peculiarities.

The anomalous location of the heart in the chest was first scientifically described in 1606 by the Italian anatomist and surgeon Jerome Fabrizius. Marco Aurelio Severino used the term "dextrocardia" in 1643 when describing a patient's mirror location of internal organs. The first coronary artery bypass surgery in a patient with dextrocardia was performed in 1980 [3].

\section{Case Report}

A 50-year-old patient was admitted to our hospital with complaints of chest pain. In 2011, this patient was implanted with a drug-eluting stent in the circumflex branch of the left coronary artery. Also in 2011, according to the results of CT, the patient was diagnosed with situs inversus totalis.

Upon admission to the clinic, the patient underwent coronary angiography, the results of which revealed stenosis of the proximal left anterior descending artery (LAD) by $60 \%$, the medial part of LAD by $70 \%$, the distal part of LAD by $70 \%$, and the proximal part of the ramus circumflexus (RCX) of the left coronary artery (LCA) by $80 \%$. Also, an echocardiogram was performed preoperatively, but no valve abnormality was detected. A computed tomography scan of the chest cavity confirmed situs inversus totalis. There were no congenital heart defects, no persistent superior vena cava, and no bilateral vena cava (Figure 1). General condition and laboratory parameters were normal.

CABG was performed promptly using 2 conduits. After medial sternotomy (Figure 2), both mammary arteries (left and right) were harvested by the skeletal method. Next, the cannulation

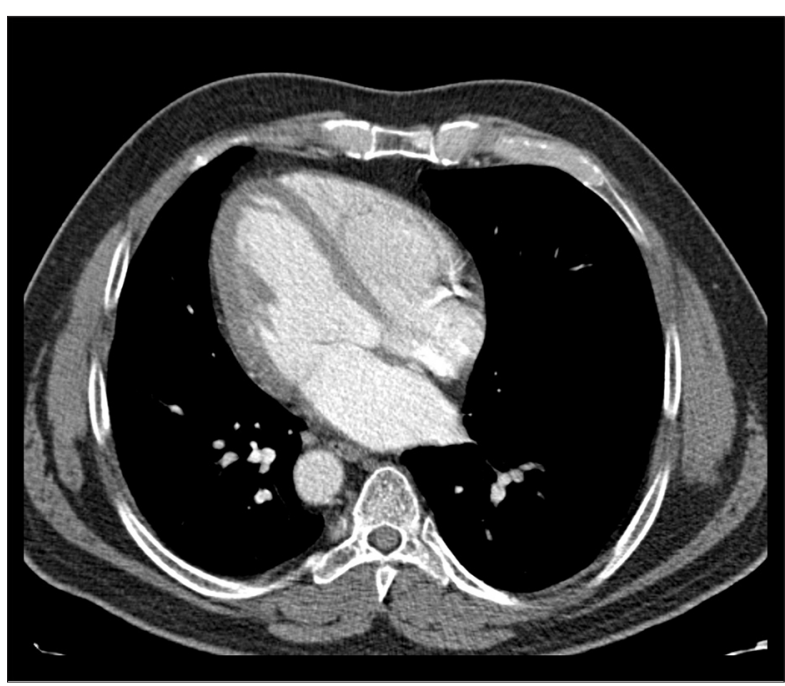

Figure 1. CT scan of the patient's chest with situs inversus totalis.

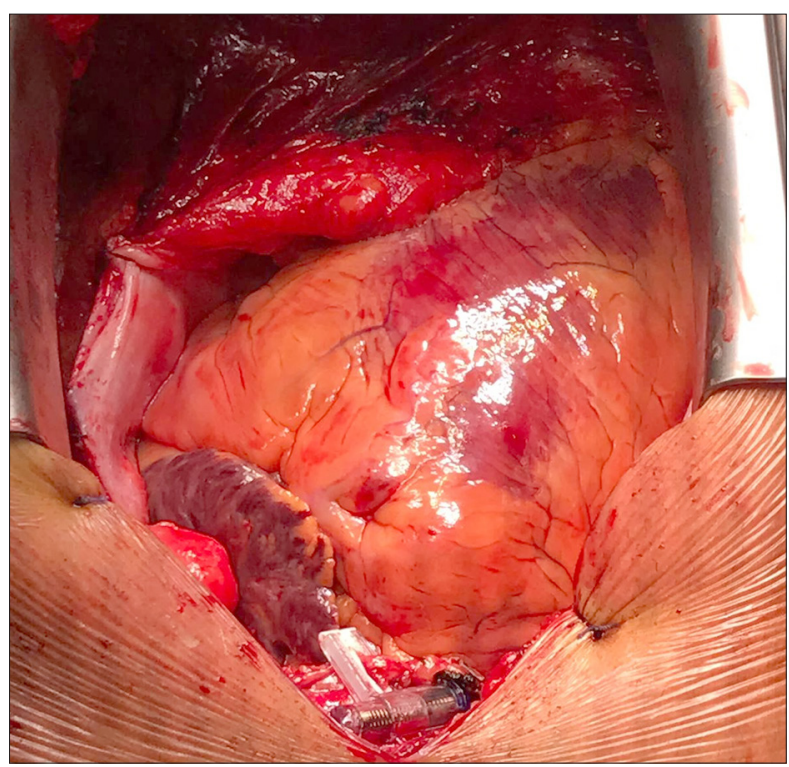

Figure 2. Patient's heart with situs inversus totalis after median sternotomy.

of the aorta and the physiologically right atrium was performed. Custodiol cardioplegia was performed antegrade in a single shot. The left internal mammary artery (LIMA) was anastomosed in situ to the LAD and the right internal mammary artery (RIMA) was anastomosed (also in situ) to the RCX (Figure 3). The surgeon, who was right-handed, was on the left side of the patient at the time of surgery. The scrub nurse was on the conventional (right) side of the patient. The aortic clamping time was 29 minutes and the cardiopulmonary bypass time was 49 minutes.

The operation was completed without complications. The patient was extubated 6 hours after surgery. On the first 


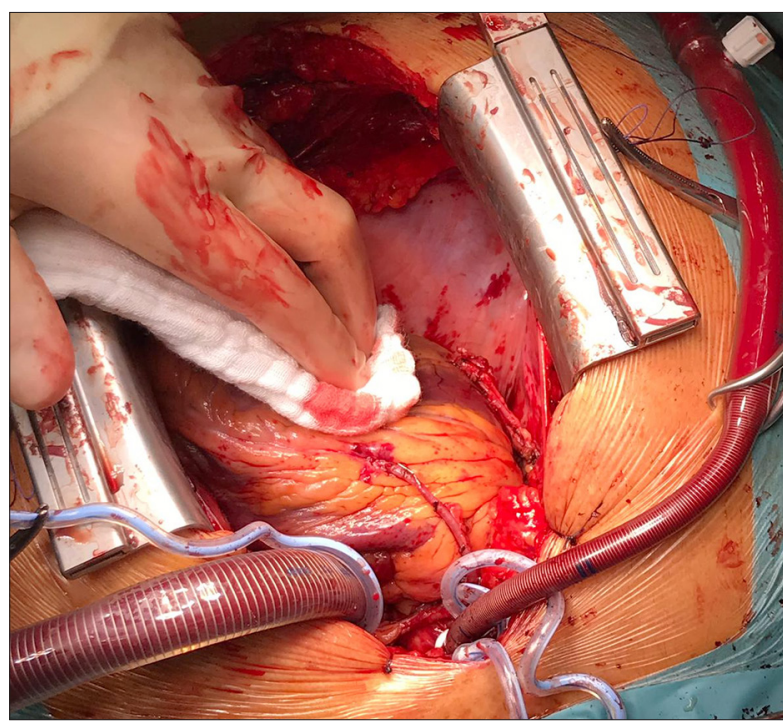

Figure 3. Patient's heart with situs inversus totalis after coronary artery bypass grafting. The left internal mammary artery (LIMA) was anastomosed to the left anterior descending artery (LAD) and the right internal mammary artery (RIMA) to the ramus circumflexus (RCX).

postoperative day, the patient was transferred to a regular ward. On day 6 after surgery, he was discharged to a rehabilitation clinic. Postoperative therapy was without complications.

\section{Discussion}

Dextrocardia is a rare congenital malformation of the cardiovascular system, in which the heart is located in the chest to the right, symmetrical to the normal location. Accordingly, all incoming and outgoing vessels of the heart are mirrored to the usual location. In the existing literature, there are isolated descriptions of the surgical treatment of coronary heart disease in patients with situs inversus totalis, many performing CABG surgery. Most surgeons in these operations stand on the left side of the patient [4]. We strongly believe that the choice of the side of the surgeon's position should be decided by the surgeon. Performing this operation is possible either on-pump or off-pump. Murtuza et al. analyzed 32 cases of patients with situs inversus totalis who underwent CABG surgery

\section{References:}

1. Deuse T, Reitz BA: Heart-lung transplantation in situs inversus totalis. Ann Thorac Surg, 2009; 88(3): 1002-3

2. Pattakos G, Chrissoheris M, Halapas A et al: Transcatheter aortic valve replacement in a patient with dextrocardia and situs inversus totalis. Ann Thorac Surg, 2019; 107(1): e33-35 from 1981 to 2009. In total, 20 patients underwent CABG with a cardiopulmonary bypass, 11 on a beating heart, 1 patient was converted to cardiopulmonary bypass intraoperatively, and in 1 case the method was not mentioned. Saphenous veins were used as a conduit in 6 cases, arterial graft in 7 cases, and both types of grafts were used in 19 cases [5].

Choosing a conduit is very important when performing a CABG operation. First of all, we decided to perform a totally arterial myocardial revascularization in a 50-year-old patient. It corresponds to current guidelines which advise avoiding the use of vein conduits in patients younger than 70 years [6]. In dextrocardia, choosing a conduit is a significant problem for the surgeon due to the abnormal position of the heart in the chest cavity. Most surgeons prefer to perform LAD anastomosis with RIMA. However, the LAD to LIMA anastomosis was also described $[7,8]$. Poncelet et al. used the gastroepiploic artery for myocardial revascularization [9]. Based on this, we can conclude that the choice of graft will depend on the location of the lesion and the number of stenotic coronary arteries.

In our case, the LAD and RCX were affected. Therefore, both RIMA and LIMA were chosen as in situ conduits because of their sufficient length for this patient, which was ascertained intraoperatively after the conduits were harvested and distally divided. The revascularization was done with anastomosis LIMA to LAD and RIMA to RCX. This choice has advantages, since the physiological blood flow is maintained by the LIMA per LAD. However, this anastomosis variant has drawbacks as well. Since the LIMA crosses the sternum from left to right, additional rethoracotomy creates additional risks of damage to the LIMA.

\section{Conclusions}

Surgical correction is indicated for many patients with dextrocardia in combination with coronary heart disease. Performing a CABG operation on these patients is challenging and requires the operating team to have certain skills. Surgeons face a number of problems and issues: preoperative preparation of the patient, the position of the surgeon to the left or right during the operation, the choice of conduit for anastomosis, and the tactics of the operation.

3. Subash S, Simha PP, Manjunatha N: Off-pump coronary artery bypass surgery in a patient with dextrocardia and situs inversus: Anesthetic, surgical consideration and role of transesophageal echocardiography. Heart Views, 2017; 18: 100-3

4. Saad RA, Badr A, Goodwin AT et al: Should you stand on the left or the right of a patient with dextrocardia who needs coronary surgery? Interact Cardiovasc Thorac Surg, 2009; 9(4): 698-702 
5. Murtuza B, Gupta P, Goli G et al: Coronary revascularization in adults with dextrocardia: Surgical implications of the anatomic variants. Texas Hear Inst J, 2010; 37(6): 633-40

6. Neumann F-J, Sousa-Uva M, Ahlsson A et al: 2018 ESC/EACTS Guidelines on myocardial revascularization. Eur Heart J, 2019; 40(2): 87-165

7. Seddio F, Colagrande L, Pellegrino A et al: [Myocardial revascularization in dextrocardia with situs inversus]. G Ital Cardiol, 1999; 29(10): 1222-26 [in Italian]
8. Baltalarli A, Tanriverdi $\mathrm{H}$, Goksin I et al: Coronary arterial revascularization in an adult with congenitally corrected transposition of great arteries and dextrocardia. J Card Surg, 2006; 21(3): 296-97

9. Poncelet AJ, Dion R, Lengele B et al: Complete arterial revascularization in coronary artery bypass grafting in a patient with solitus inversus totalis. J Cardiovasc Surg (Torino), 2006; 47(4): 477-79 